\title{
PROCESSOS DE GESTÃO dO CONHECIMENTO NO ENSINO SUPERIOR: ESTUDO EM UMA UNIVERSIDADE DE SANTA CATARINA
} KNOWLEDGE MANAGEMENT PROCESSES: STUDY AT A UNIVERSITY OF SANTA CATARINA

Recebido em 20.09.2016. Aprovado em 05.09.2017

Avaliado pelo sistema double blind review

DOI: http://dx.doi.org/10.12712/rpca.v11i4.823

\section{Carla de Cássia Nardelli Vieira}

Universidade Regional de Blumenau (FURB), Blumenau/SC, BRASIL

carla@furb.br

\section{Carolina Klein Padilha}

Universidade Federal de Santa Catarina (UFSC), Florianópolis/SC, BRASIL

carolakl@terra.com.br

\section{Denise Del Prá Netto Machado}

Universidade do Sul de Santa Catarina (UNISUL), Florianópolis/SC, BRASIL

profadenisedelpra@gmail.com

\section{Luciano de Castro Carvalho}

Universidade Regional de Blumenau (FURB), Blumenau/SC, BRASIL

luccar@gmail.com

\section{Resumo}

Este estudo buscou analisar a percepção dos colaboradores de uma instituição de ensino superior quanto à eficácia dos processos de gestão do conhecimento em níveis individual, de grupo e organizacional. Realizouse pesquisa quantitativa, descritiva transversal, do tipo survey. Para a coleta de dados utilizou-se questionário fechado, elaborado e validado conforme estudo de Sabherwal e Becerra-Fernandez (2003). Para a análise dos dados empregou-se estatística e modelagem de equações estruturais. Percebeu-se que na dimensão ontológica a percepção de aprendizagem é originada em nível individual, transferida para o grupo e para a organização. $\mathrm{Na}$ dimensão epistemológica a espiral do conhecimento é iniciada com a conversão de conhecimento tácito pela socialização e externalização. Concluiu-se que o conhecimento é gerado e também socializado nos níveis de aprendizagem.

Palavras-chave: Gestão do conhecimento. Aprendizagem organizacional. Instituição de Ensino Superior.

\begin{abstract}
This study aimed to analyze the perception of employees of a university on the effectiveness of knowledge management processes in individual, group and organizational levels. It is a quantitative, transversal, descriptive and survey research. For data collection was used closed questionnaire developed and validated according to Sabherwal and Becerra-Fernandez (2003) study. For data analysis was used statistical and structural equation modeling. It was noticed that at ontological dimension the perception of learning originates at the individual level, transferred to the group and the organization. In epistemological dimension knowledge spiral starts with the conversion of tacit knowledge through socialization and externalization. It was concluded that knowledge is generated and socialized in learning level.
\end{abstract}

Keywords: Knowledge management. Organizational learning. University. 


\section{Introdução}

O conhecimento organizacional pode ser armazenado de várias formas, incluindo a mente humana, documentos, políticas e procedimentos e compartilhado entre os indivíduos por meio de conversas, treinamentos, programas de aprendizagem e relatórios (SABHERWAL; BECERRAFERNANDEZ, 2003). Mas o conhecimento é criado pela aprendizagem dos indivíduos por meio de suas percepções e posteriormente partilhado e combinado com outros conhecimentos. A valorização do conhecimento para as organizações fica evidente na sociedade contemporânea, quando é discutida por diversos estudiosos sobre a gestão deste ativo intangível, como Choo (2003), Isidro-Filho (2009), Argote e Miron-Spektor (2011), Hartung e Oliveira (2013), Amayah (2013), Pokharel e Choi (2015), entre outros.

Terra (2005) explica gestão do conhecimento como a organização das principais políticas, processos e ferramentas gerenciais e tecnológicas das organizações, a fim de compreender os processos de geração, identificação, validação, disseminação, compartilhamento e uso dos conhecimentos individuais e coletivos para gerar resultados e benefícios para a organização e seus colaboradores.

As instituições de ensino superior (IES), por sua própria natureza e atividade, são ambientes de compartilhamento e criação de conhecimento. São consideradas organizações complexas por possuírem características peculiares que as distinguem das demais organizações. Aspectos como a multiplicidade de objetivos, o tipo de profissional que nela atua e o fato de estarem voltadas para o ensino, a pesquisa e a extensão fizeram com que este tipo de organização desenvolvesse um estilo próprio de estrutura (SILVA, 1991).

Supõe-se que o conhecimento gerado, não somente por pesquisas e trabalhos acadêmicos, mas também aquele criado por todos os colaboradores destas instituições devem propiciar a melhoria de seus processos de gestão. Estas organizações contribuem na transformação da sociedade em sociedade do conhecimento. Neste sentido, a gestão do conhecimento vem ao encontro dos objetivos das IES ao possibilitar a sistematização e disponibilização a todos os participantes do processo de dinamização do capital intelectual, novas fontes de saber para a realização de suas atividades. A gestão do conhecimento absorve a função de dispor ferramentas, que reserva um lugar privilegiado ao conhecimento e à sua criação.
Dessa forma, o objetivo deste estudo é analisar a percepção dos colaboradores de uma IES quanto à eficácia dos processos de gestão do conhecimento para propiciar a aprendizagem, que leva à criação de conhecimento. Tem-se como pergunta de pesquisa: Quais processos de gestão do conhecimento são perceptíveis aos seus colaboradores de uma instituição de ensino superior como facilitadores para a aprendizagem organizacional?

Neste sentido, serão observados os tipos de aprendizagem e o processo de criação do conhecimento, baseados nos estudos de Sabherwal e Becerra-Fernandez (2003). O modelo desenvolvido por Sabherwal e Becerra-Fernandez (2003) aborda autores da literatura, como Nonaka (1994), Nonaka e Takeuchi (1995), Daft e Weick (1984), Fiol (1994), Starbuck e Milliken (1988) e Probst, Büchel e Raub (1998). Contempla a mensuração da percepção da eficácia dagestão do conhecimento (em nívelindividual, de grupo e organizacional) e a mensuração dos processos de gestão do conhecimento (externalização, combinação, socialização e internalização).

\section{Gestão do conhecimento}

Gestão do conhecimento é definida por Terra (2005) como um complexo processo associado à própria evolução da teoria organizacional e dependente de análise profunda da relação entre as variáveis: ambiente econômico e social, evolução tecnológica, lógica organizacional e concepções sobre a natureza humana. Trata-se de uma ferramenta para administrar a informação e distribuí-la de forma a ser transformada em conhecimento, de modo a ser subsídio para a tomada de decisão. Para compreensão do significado destes conceitos serão explanados conceitos sobre aprendizagem, conhecimento, processos de criação do conhecimento e como eles se inter-relacionam em IES.

De acordo com Hartung e Oliveira (2013) a gestão do conhecimento é uma estrutura que pode auxiliar os administradores no tratamento de ativos intelectuais, estimulando ações e comportamentos que possibilitam o reconhecimento do conhecimento necessário para a organização, proporcionando que este seja adquirido, criado, compartilhado e utilizado para atingir os objetivos da organização. Os autores destacam que a gestão do conhecimento pode ser uma forma pela qual as empresas possam reconhecer os conhecimentos necessários para maximizar a produtividade e a eficiência em longo prazo. 
Argote e Miron-Spektor (2011) discorrem que o conhecimento organizacional é formado por experiências, valores e informações contextualizadas, como também por interpretação e julgamento do sujeito, que possibilitam a manifestação de mudanças cognitivas, que podem incluir componentes explícitos e tácitos, incluindo os indivíduos e as rotinas organizacionais.

No entanto, de acordo com He, Baruch e Lin (2014) a disponibilidade de conhecimento organizacional não assegura sua utilização pelos indivíduos da organização, pois por vezes os conhecimentos necessários para diversas atividades podem não serem percebidos e identificados pelos indivíduos. Nesse sentido, Hartung e Oliveira (2013) comentam que a utilização é o conjunto de ações que proporciona a aplicação do conhecimento em consonância com os objetivos da organização.

De acordo com Cardoso e Gomes (2011) o compartilhamento do conhecimento pode ocorrer de modo intencional ou não intencional, sendo que a forma intencional se dá quando a ação ocorre de forma consciente, ou seja, por meio de atitudes explícitas e arquitetadas, como reuniões de trabalho e ações de treinamento Já a forma não intencional pode ocorre em conversas nas pausas do trabalho e também por meio de histórias contadas sobre a realização de tarefas passadas.

Para Hartung e Oliveira (2013) o compartilhamento do conhecimento é um conjunto de ações que propicia o intercâmbio de conhecimento através de interações sociais em ambientes físicos ou virtuais, de forma que ocorra o entendimento do conhecimento compartilhado. Ramayah, Yeap e Ignatius (2014) destacam que o compartilhamento do conhecimento deve ser foco central da discussão que envolve o entendimento da gestão do conhecimento como ação organizacional que possibilita a utilização do conhecimento em benefício dos objetivos organizacionais. Pokharel e Choi (2015) discorrem que para que ocorra o compartilhamento do conhecimento é fundamental que sejam facilitadas situações rotineiras de aprendizagem.

Segundo Fialho et al. (2006), a aprendizagem pode ser definida como uma das principais fontes de recursos de uma organização, de modo a obter vantagem competitiva. A gestão do conhecimento dentro da organização tem por objetivo acelerar a aquisição do conhecimento sobre mercado, tendências, produtos, concorrência, a fim de diminuir riscos e aumentar a competitividade. Fialho et al. (2010) ainda argumentam que a aprendizagem é um processo de conflito que ocorre por meio da interação entre as pessoas e o ambiente, envolvendo observação, reflexão, experiências concretas, que geram uma permanente revisão dos conceitos aprendidos.

A aprendizagem individual, de acordo com Fialho et al. (2010) refere-se ao modo como o indivíduo assimila um novo dado ou informação, reflete sobre experiências passadas, chega a uma conclusão ou à concepção de um novo conhecimento e age para atingir um objetivo. Os modelos individuais de aprendizagem evoluem para modelos com maior nível de organização, convergindo as ações da empresa para o aprendizado em equipe.

A interação social e com o ambiente são essenciais para o desenvolvimento do aprendizado. A aprendizagem em equipe diz respeito ao trabalho em grupo em uma organização, em estabelecer nova organização de tarefas, novas ferramentas de acompanhamento de resultados, novas maneiras de avaliar o sucesso da gestão e dos processos de operação. Isto acontece devido à comunicação estabelecida entre os indivíduos, que possibilita a criação e recriação da organização e esta, por sua vez, influência grupos e seu contínuo processo de recriação e aprendizagem (FIALHO et al., 2010).

Por sua vez, a aprendizagem organizacional significa a transferência da aprendizagem das equipes para toda a organização, por meio da explicitação dos conhecimentos adquiridos (FIALHO et al., 2006). A aprendizagem organizacional, de acordo com Sabherwal e Becerra-

Fernandez (2003) transcende a aprendizagem de cada membro da organização, mas ocorre por meio dele, com resultados positivos e negativos que enfrentam a partir de seus comportamentos. $\mathrm{O}$ valor dessa aprendizagem experimental dependerá da medida em que cada indivíduo aprende, bem como a aprendizagem individual fica internalizada na memória organizacional. Sabherwal e Becerra-Fernandez (2003) relatam que a aprendizagem também acontece interorganizacional, porém não será abordada neste estudo.

A aprendizagem e o conhecimento estão interligados sistemicamente. Para Sanchez e Heene (1997), conhecimento pode ser entendido como um conjunto 
de crenças e modelos mentais nos quais são geradas as decisões. Assim, o conhecimento organizacional é estabelecido pelo compartilhamento dessas crenças, representando a coletividade da organização. Já a aprendizagem é o processo no qual o conhecimento é gerado, por meio de diferentes fontes de dados e informações compreendidas pelas experiências individuais e coletivas (COHEN; LEVINTHAL, 1990).

A aprendizagem organizacional é percebida pelos processos de ação, reflexão e disseminação do conhecimento dos colaboradores da organização, nos quais os resultados são relacionados à interpretação e à geração de conhecimentos e competências, mesmo em casos negativos (ZANGISKI; LIMA; COSTA, 2009. Para Nonaka e Takeuchi (2008), o processo de transformação do conhecimento criado pelos indivíduos para os níveis de grupo e organizacional representa a dimensão ontológica da criação do conhecimento, sendo dependentes entre si, relacionando-se de forma iterativa e contínua. Porém salientam que em sentido rígido, o conhecimento somente é criado pelos indivíduos, os quais são apoiados pelas organizações com contextos que propiciem a amplificação deste conhecimento para que se cristalize como parte da rede de conhecimentos da organização.

Figura 1. Criação do conhecimento pelas dimensões epistemológica e ontológica

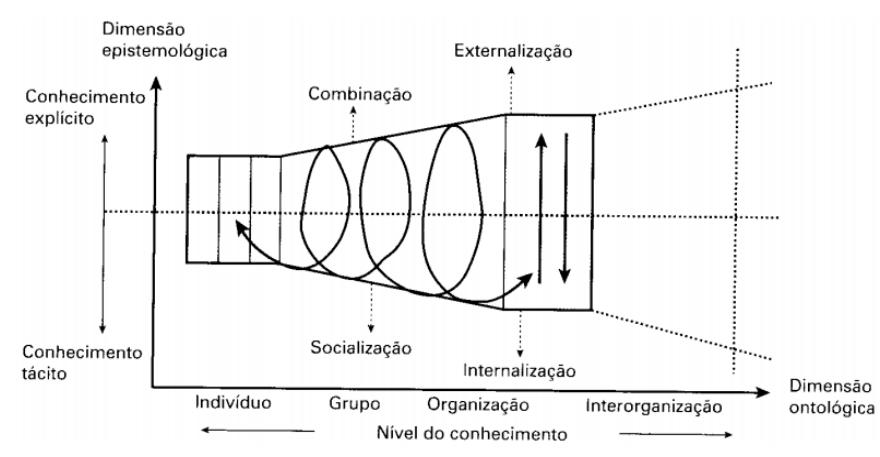

Fonte: Nonaka e Takeuchi (2008, p. 70)

O conhecimento decorre da informação e esta de um conjunto de dados. A partir desta definição, o conhecimento pode ser interpretado como informações transformadas pela ação dos indivíduos ou derivado de outros conhecimentos, que alinhados e sobrepostos podem lançar olhares para diferentes perspectivas de situações corriqueiras
(DAVENPORT; PRUSAK, 1998; TERRA, 2005). Conhecimento também é interpretado por Sabherwal e Becerra-Fernandes (2003) como um conjunto de crenças justificadas que melhoram a capacidade de uma entidade para uma ação efetiva. Esta definição incorpora o conhecimento explícito e o tácito, que, para Nonaka e Takeuchi (2008), compõe a dimensão epistemológica da criação do conhecimento.

O conhecimento explícito é o objetivo final da espiral do conhecimento e pode ser expresso em números e palavras compartilhadas formal e sistematicamente na forma de dados, especificações, manuais, entre outros. Já o conhecimento tácito, que inclui ideias, intuições e palpites, é inerente ao indivíduo, difícil de ser expresso, formalizado e compartilhado, pois depende das experiências vividas e das percepções cognitivas (NONAKA, 2000; SABHERWAL; BECERRA-FERNANDEZ， 2003; NONAKA; TAKEUCHI, 2008).

Terra (2001), considerando que a principal vantagem das organizações está no conhecimento tácito dos indivíduos, relata que a gestão do conhecimento resulta na realização de ações gerenciais que reúnam esforços nos planos organizacionais e individuais e estratégicos e operacionais, propondo que estas atividades de gestão proporcionem o compartilhamento de conhecimento (AMAYAH, 2013).

Baseado no estudo de Terra (2001), Heizig (2009) aponta quatro dimensões relevantes para o processo de implantação da gestão de conhecimento, sendo: dimensão humana, em que são expostos aspectos culturais, de liderança e de relacionamento entre as próprias pessoas; dimensão organizacional, em que emergem aspectos estruturais e processuais da organização; dimensão tecnológica, na qual são tratados os meios tecnológicos disponíveis para a implantação da gestão; e a dimensão dos processos, em que são apresentados aspectos de estratégias e controle usados pela organização.

Os processos de criação do conhecimento são abordagens amplas, no qual o conhecimento é descoberto, capturado, compartilhado ou aplicado (SABHERWAL;

BECERRA-FERNANDEZ, 2003). Segundo Nonaka e Takeuchi (1995) e Batista (2012) a criação do conhecimento diz respeito a um conjunto de ações que possibilitam a criação de novos conhecimentos, resultantes da interação entre os sujeitos, passando do nível do indivíduo para a mais alta esfera organizacional. 
Nonaka e Takeuchi (1997) identificaram quatro processos de conversão do conhecimento, decorrentes da interação do conhecimento nas dimensões epistemológica e ontológica: internalização (de explícito para tácito); externalização (de tácito para explícito); socialização (de tácito para tácito); e combinação (de explícito para explícito).

Figura 2 . Processos de gestão do conhecimento

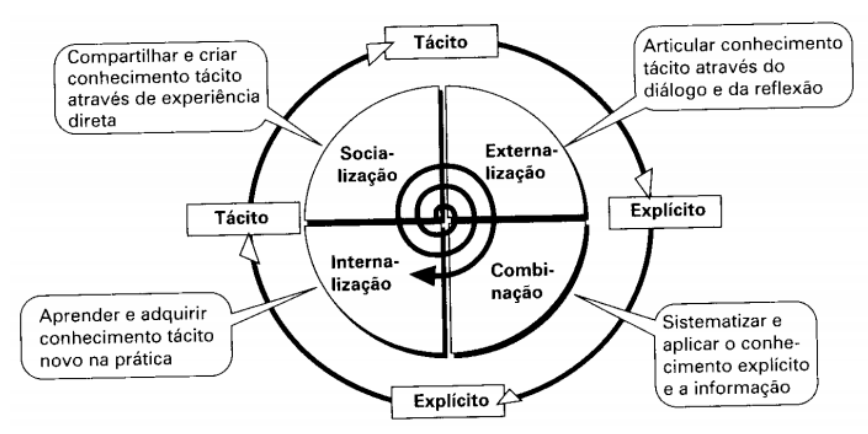

Fonte: Takeuchi e Nonaka (2008, p. 24)

A internalização é o processo que auxilia a conversão do conhecimento explícito em tácito. O conhecimento explícito pode ser incorporado na ação e prática de modo individual, em situações virtuais, lendo ou ouvindo histórias ou experimentalmente por meio de simulações ou experimentos. Em contraponto, a externalização envolve a expressão do conhecimento tácito e sua tradução em formas compreensíveis para que outros possam entender. A externalização conta com técnicas para expressar ideias ou elementos como palavras, conceitos, imagens, linguagem figurada, raciocínio indutivo/dedutivo ou inferência criativa e desta forma permite a tradução do conhecimento pessoal ou profissional em formas explícitas que são fáceis de entender (NONAKA; TAKEUCHI, 1997; SABHERWAL; BECERRA-FERNANDEZ, 2003).

A socialização, segundo Nonaka e Takeuchi (1997), envolve a partilha do conhecimento tácito entre os indivíduos, pela troca de experiências em atividades conjuntas, em vez de forma escrita ou verbal. Ao transferir ideias e imagens, permite aos recémchegados a possibilidade de perceberem a forma como os outros pensam e sentem. O conhecimento é produzido em um ambiente de grupo, por meio da partilha do conhecimento comum e da sinergia dos indivíduos. Os processos sociais desempenham papel relevante na transição do conhecimento de nível individual para equipe, porém ao depender da proximidade física e da ação conjunta, desempenha um papel discreto para a partilha de conhecimento no nível organizacional (SABHERWAL; BECERRAFERNANDEZ, 2003).

Para Sabherwal e Becerra-Fernandez (2003), o processo de combinação envolve a conversão do conhecimento explícito em novos conjuntos de conhecimento explícito, concentrando-se em comunicação, difusão, integração, combinação e sistematização do conhecimento, contribuindo para a aprendizagem do grupo e da organização. A combinação ajuda a integrar os conhecimentos dos membros do grupo, mas o novo conhecimento gerado pela combinação, muitas vezes transcende o grupo.

Conforme Nonaka e Takeuchi (2008) o processo de criação do conhecimento inicia-se pela socialização, com a criação de um campo de interação, que facilita o compartilhar das experiências e modelos mentais dos membros. A externalização é desencadeada pelo diálogo e reflexão coletiva significativos, com a articulação do conhecimento oculto. A combinação é disparada pela rede de conhecimento recentemente criado e do conhecimento existente de outras seções da organização, cristalizando-os dessa maneira em um novo produto, serviço ou sistema administrativo. Por fim, o aprender fazendo desencadeia a internalização.

O conteúdo gerado por cada processo é diferente, a socialização propicia o compartilhamento do conhecimento por meio de conversas informais. A externalização produz o conhecimento conceitual, na qual ideias são expressas por meio de metáforas, tornando uma diretriz para a criação do conhecimento sistêmico. A combinação gera o conhecimento sistêmico, resultando novos conceitos, tecnologias, processos e protótipos. E, por fim, a internalização produz o conhecimento operacional e, muitas vezes baseado na experiência, origina um novo ciclo de criação do conhecimento (NONAKA; TAKEUCHI, 1997).

Sabherwal e Becerra-Fernandez (2003) argumentam que as organizações não têm cérebros, mas sistemas cognitivos e memórias. Por meio dos indivíduos, que desenvolvem suas personalidades, hábitos pessoais e crenças ao longo do tempo, as organizações desenvolvem visões do mundo e ideologias. Colaboradores entram e saem, mudança de lideranças 
ocorrem, mas as memórias das organizações preservam certos comportamentos, mapas mentais, normas e valores ao longo do tempo.

Segundo Etzioni (1989), a universidade pode ser caracterizada como organização especialista, uma vez que favorece a geração, utilização e preservação do conhecimento. Este tipo de organização caracteriza-se pela ênfase na busca dos seus objetivos, pelo número expressivo de especialistas (professores) em relação aos não especialistas e pela relação de autoridade que existe entre eles.

A educação superior, de acordo com a Lei de Diretrizes e Bases (LDB), art. 43, tem a finalidade de: estimular a criação cultural e o desenvolvimento do espírito científico; formar profissionais aptos para a inserção e participação no desenvolvimento da sociedade; incentivar a pesquisa e a investigação científica, visando o desenvolvimento da ciência e tecnologia; promover conhecimentos culturais científicos e técnicos e comunicar o saber através de publicações e outras formas de comunicação; suscitar o desejo de aperfeiçoamento permanente; estimular o conhecimento dos problemas do mundo e estabelecer com a sociedade uma relação de reciprocidade; e promover a extensão aberta à participação da população, visando a difusão das conquistas e benefícios resultadas da criação do saber. Ainda na LDB, o ensino superior abrange os cursos sequenciais, de graduação, de pós-graduação e extensão.

A Lei ${ }^{\circ} 5540 / 68$, determina a estrutura organizacional das IES, com as seguintes características citadas por Machado e Silveira (1998): possuir unidade de patrimônio e administração; ter estrutura orgânica com base em departamentos reunidos ou não em unidades mais amplas; ter unidades de ensino e pesquisa; e possuir racionalidade de organização. De acordo com os autores, por meio destas características, as IES adotaram como órgãos superiores deliberativos os Conselhos Universitários, de Curadores e de Ensino, Pesquisa e Extensão e, como órgão superior executivo a Reitoria, as Pró-Reitorias, as Diretorias de unidade administrativas e os órgãos de apoio.

As universidades por desempenharem tarefas de ensino, pesquisa e extensão se diferenciam dos demais tipos de organização e são consideradas complexas pela imposição dos métodos de trabalho diferentes, pela natureza dos trabalhos, pelas tecnologias utilizadas pelas pessoas que trabalham na organização e por seus clientes. Meyer Junior (2007) e Pereira et.al.
(2008) destacam as principais características das IES: metas difusas e ambíguas; clientes com necessidades distintas e complexas, o que gera problemas de tecnologia; predominância de pessoal especializado; vulnerabilidade ao ambiente externo; natureza intangível de seus produtos; processo decisório; dificuldade de avaliar o valor agregado; interesses organizacionais sobrepujados pelos interesses profissionais individuais; e administração universitária.

A gestão de uma instituição de ensino típica é formada por um conjunto de decisões assumidas a fim de obter um equilíbrio dinâmico entre missão, objetivos, meios e atividades acadêmicas e administrativas. E um modelo de gestão do conhecimento desenvolvido para este tipo de organização busca desenvolver estratégias específicas para incentivar as transferências do conhecimento de forma a gerar mais conhecimento, facilitar o trabalho de seus colaboradores e aumentar a competitividade da organização (CARDOSO; MACHADO, 2008; CAJUEIRO; SICSÚ; RIBEIRO, 2009).

\section{Procedimentos metodológicos}

Para a elaboração desta pesquisa, utilizou-se a pesquisa quantitativa, descritiva transversal, do tipo levantamento de campo ou survey. Para a coleta de dados foi utilizado questionário fechado, elaborado e validado conforme estudos de Sabherwal e BecerraFernandez (2003). A tradução do instrumento foi realizada com o auxílio de ferramentas de tradução e dicionário do idioma da língua inglesa. Após esta etapa, o questionário foi validado pelos autores deste artigo.

A pesquisa quantitativa é caracterizada pela descrição numérica de tendências, atitudes e opiniões de uma população ao se estudar uma parcela dela (CRESWELL, 2007). A pesquisa descritiva é estruturada para medir características descritivas em uma questão de pesquisa, cujo corte transversal acontece pela coleta de dados em um ponto do tempo e sintetizados estatisticamente. Levantamento de campo ou survey é caracterizado pela interrogação direta das pessoas e apresenta como vantagens o conhecimento direto da realidade, economia, rapidez na obtenção dos dados e quantificação (HAIR JR. et al., 2005).

O questionário foi formulado com 24 questões, estruturado em três blocos: perfil dos respondentes, 
aprendizagem organizacional e processos de gestão do conhecimento. Cada respondente apontou seu grau de concordância, em uma escala intervalar de 7 pontos, que variou de "Discordo totalmente" (1) a "Concordo totalmente" (7). O instrumento de coleta de dados foi aplicado aos colaboradores técnico-administrativos e docentes de uma instituição de ensino superior do Vale do Itajaí (SC), no período de 20 a 31 de março de 2014.

Vale lembrar que a adaptação do questionário desenvolvido por Sabherwal e Becerra-Fernandez (2003) manteve os mesmos construtos. Assim, a estrutura a ser verificada na IES pesquisada contempla questões sobre aprendizagem individual, de grupo e organizacional, socialização, externalização, combinação e internalização. Para o relacionamento entre as dimensões foram propostas nove hipóteses, cujo modelo simplificado está exposto na Figura 3.

Figura 3 . Estrutura da modelagem por equações estruturais

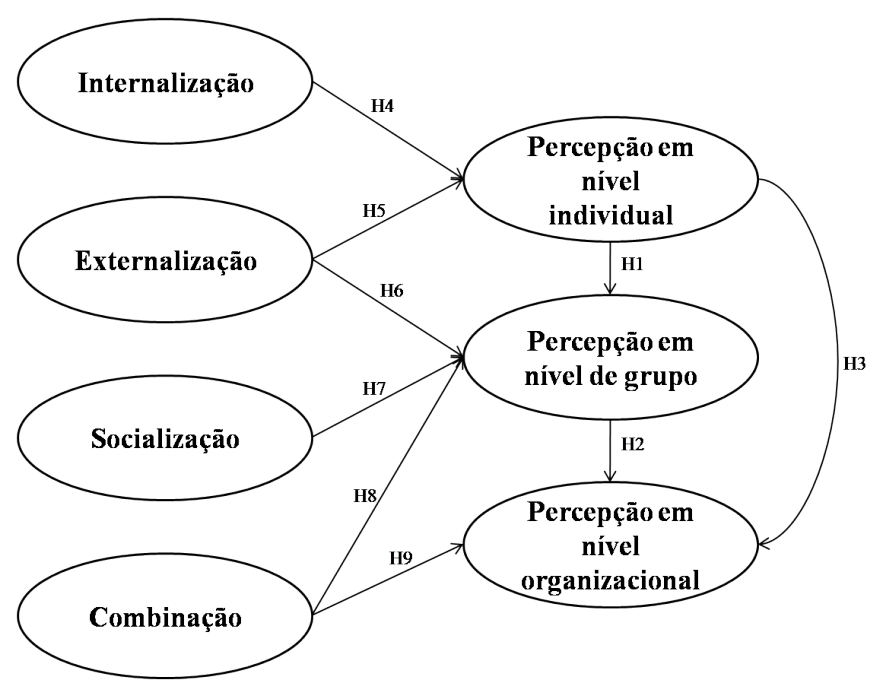

Fonte: Sabherwal e Becerra-Fernadez (2003).

As hipóteses desenvolvidas foram:

H1: A percepção da eficácia da GC no nível individual facilita a percepção da eficácia da GC no nível de grupo.

H2: A percepção da eficácia da GC no nível de grupo facilita a percepção da eficácia da GC no nível organizacional.

H3: A percepção da eficácia da GC no nível individual facilita a percepção da eficácia da GC no nível organizacional.

H4 : A internalização facilita a percepção da eficácia GC em nível individual.

H5 : A exteriorização facilita a percepção da eficácia GC em nível individual.

H6 : A exteriorização facilita a percepção da eficácia GC em nível em nível do grupo.

H7: A socialização facilita a percepção da eficácia GC em nível do grupo.

H8 : A combinação facilita a percepção da eficácia GC em nível do grupo.

H9 : A combinação facilita a percepção da eficácia GC em nível organizacional.

A população diz respeito à totalidade de elementos que possuem um conjunto de características comuns e pode se referir a um grupo de pessoas que se pretende conhecer, como também a países, fatos sociais, produção agroindustrial, empresas públicas ou privadas, entre outros (HAIR JR. et al., 2005). A população é formada por 1.524 colaboradores de uma instituição de ensino superior do Vale do Itajaí (SC), cuja amostra foi obtida a partir de 257 respondentes, representando $16,9 \%$ da população, conforme pode ser observado na Tabela 1.

Tabela 1 . Composição da amostra

\begin{tabular}{|l|c|c|c|}
\hline Função & População & Respondentes & $\begin{array}{c}\text { Amostra } \\
\text { Final }\end{array}$ \\
\hline Docentes & 864 & 118 & $13,7 \%$ \\
\hline $\begin{array}{l}\text { Técnico- } \\
\text { administrativos }\end{array}$ & 660 & 139 & $21,1 \%$ \\
\hline TOTAL & $\mathbf{1 . 5 2 4}$ & $\mathbf{2 5 7}$ & $\mathbf{1 6 , 9 \%}$ \\
\hline
\end{tabular}

Fonte: dados da pesquisa.

Após a coleta de dados partiu-se para análise e a interpretação dos dados, as quais visam respectivamente organizar os dados a fim de se reportar ao problema proposto e buscar respostas de maneira ampla por meio da interação dos conhecimentos obtidos (HAIR JR. et 
al., 2005). Para esta etapa, os dados foram tabulados em planilha eletrônica por meio do software Excel ${ }^{\circledR}$. As perguntas foram agrupadas às dimensões as quais pertencem. A base de dados contida na planilha do Excel foi importada pelo software estatístico SPSS (Statistical Package for the Social Sciences).

A primeira análise realizada foi a de frequência, pela qual se pode evidenciar as respostas que apontaram as dimensões percebidas pelos colaboradores. Este procedimento foi realizado com as questões do instrumento de coleta de dados, agrupadas em dimensões. A segunda análise buscou verificar se a estrutura proposta por Sabherwal e BecerraFernandez (2003) conforme a figura 3, poderia ser observada na IES pesquisada. Para isto, fez-se uso da técnica de modelagem de equações estruturais por meio do módulo AMOS, empregando as dimensões percebidas pelos colaboradores. Este software permite gerar as equações de correlação entre as dimensões, demonstrando graficamente o valor de impacto e os valores para explicação do modelo.

\section{Análise dos resultados}

Este capítulo traz a apresentação e discussão dos dados apurados. Ressalta-se que os resultados obtidos refletem o perfil de comportamento dos colaboradores da IES em que a pesquisa foi aplicada. Inicialmente é caracterizada a instituição em que a pesquisa foi aplicada e em seguida é relatado o perfil da amostra, por meio da identificação dos respondentes. Posteriormente, faz-se a apresentação e a análise das respostas que medem a percepção dos processos de gestão do conhecimento que facilitam a aprendizagem organizacional.

\section{Caracterização da instituição}

A IES pesquisada é incluída como órgão autônomo na estrutura administrativa do Poder Executivo Municipal, uma instituição oficial de direito público. Sua trajetória conta com mais de 50 anos a partir da iniciativa da comunidade do interior de Santa Catarina, que almejava a presença de uma universidade na região. A instituição conta com aproximadamente 50 cursos de graduação, 11 mestrados recomendados pela CAPES, 3 doutorados, além de ensino médio, especializações e cursos de extensão para a comunidade acadêmica e externa, nos quais estão matriculados em torno de 12 mil alunos. Está consolidada como um dos principais polos de conhecimento do Sul do Brasil graças à qualidade de seu ensino, pesquisa e atividades de extensão. Sua estrutura organização é composta por: órgãos de deliberação coletiva: o conselho universitário e o conselho de ensino, pesquisa e extensão; órgão executivo (Reitoria), os quais são subordinados as pró-reitorias, procuradoria geral, controladoria geral, ouvidoria geral, coordenadorias de gestão superior e órgãos suplementares; unidades universitárias e unidade de ensino médio e profissionalizante.

Os objetivos organizacionais da Instituição são descritos como: Missão: promover o ensino, a pesquisa e a extensão, fomentando o desenvolvimento socioeconômico sustentável e o bem-estar social; Visão: ser uma Universidade pública, reconhecida pela qualidade da sua contribuição na vida regional, nacional e global; Valores: comprometimento: com a transparência nos processos e atos institucionais; com a participação democrática das comunidades interna e externa nas políticas e decisões institucionais; com a valorização dos discentes e dos servidores; com a formação integral do ser humano; com a democracia, com a ética e com a pluralidade; com o desenvolvimento social e sustentável; com a manutenção da sua identidade e tradição; com respeito à natureza e a todas as formas de vida; efetividade: como universidade pública; na socialização do conhecimento e de tecnologias; na indissociabilidade entre ensino, pesquisa e extensão; na integração e inserção comunitária; inovação: nos processos de humanização; nos processos de ensinoaprendizagem, de pesquisa e extensão; nas soluções para atendimento às demandas da sociedade; nos processos de internacionalização; na gestão acadêmica e administrativa.

\section{Perfil da amostra}

Foram obtidos 257 questionários respondidos, representando $16,9 \%$ da população. A primeira parte do questionário objetivou estabelecer o perfil dos respondentes quanto à idade, ao gênero, à escolaridade e à função na Instituição.

Quanto à idade percebeu-se que 30\% dos respondentes possuem idade entre 36 e 45 anos, seguidos dos que têm idade entre 46 e 55 anos, com $26,8 \%$. Os respondentes com idade entre 18 e 25 anos representam $8,8 \%$ do total e aqueles que estão entre 26 e 35 anos, correspondem a 21,6\%. Aqueles que têm idade superior a 56 anos representam 6,4\%. 
Quanto ao gênero, há equilíbrio entre os respondentes, com 47,2\% do gênero feminino e 52,8\% do gênero masculino. Quanto à escolaridade, a maioria dos colaboradores possui graduação $(22,0 \%)$ ou pós-graduação (72,4\%). No que diz respeito à função exercida na instituição, $45,2 \%$ dos respondentes são professores e $54,8 \%$ são técnico-administrativos.

\section{Resultados das dimensões}

Inicialmente foram observadas as dimensões sobre aprendizagem individual, em grupo e organizacional. As análises dos dados foram obtidas pela frequência das respostas. Os resultados podem ser observados nas Tabelas 2.

Tabela 2 . Frequências de percepção da aprendizagem

\begin{tabular}{|l|c|c|c|c|c|c|c|c|c|c|}
\hline \multicolumn{1}{|c|}{ Escala } & $\mathbf{1}$ & $\mathbf{2}$ & $\mathbf{3}$ & $\mathbf{4}$ & $\mathbf{5}$ & $\mathbf{6}$ & $\mathbf{7}$ & Discordo & Neutro & Concordo \\
\hline Individual & 7 & 38 & 59 & 152 & 200 & 179 & 136 & $\mathbf{1 3 , 5}$ & $\mathbf{1 9 , 7}$ & $\mathbf{6 6 , 8}$ \\
\hline Em grupo & 20 & 56 & 85 & 169 & 176 & 149 & 116 & $\mathbf{2 0 , 9}$ & $\mathbf{2 1 , 9}$ & $\mathbf{5 7 , 2}$ \\
\hline Organizacional & 60 & 112 & 160 & 209 & 142 & 61 & 27 & $\mathbf{4 3 , 1}$ & $\mathbf{2 7 , 1}$ & $\mathbf{2 9 , 8}$ \\
\hline
\end{tabular}

Fonte: dados da pesquisa.

A percepção mais acentuada sobre os processos de aprendizagem foi no nível individual com 66,8\%, seguida do nível em grupo com $57,2 \%$. Somente a aprendizagem organizacional obteve maior discordância, com $29,8 \%$ de concordância e 43,1\% de discordância. Estes resultados refletem as conclusões de Sabherwal e BecerraFernandez (2003), as quais evidenciam que a aprendizagem organizacional depende das demais aprendizagens, assim como a aprendizagem em grupo é dependente da aprendizagem individual.

Para os professores a aprendizagem individual obteve maior média $(5,19)$ em relação aos servidores técnicosadministrativos (4,91). A característica da atividade docente é relacionada com o compartilhamento dos conhecimentos com seus discentes, no entanto sua dependência da organização ou do grupo para sua atuação é reduzida, mas a aprendizagem individual é ressaltada. Assim a disponibilidade de conhecimento oferecida pela organização mostra-se suficiente para o exercício de sua atividade.

Por outro lado, os servidores técnicos-administrativos necessitam de maior interação do grupo para balizarem suas atividades profissionais. Esta situação é constatada pela diferença das médias entre docentes $(4,55)$ e técnicos-administrativos $(4,85)$. A interação entre os professores acontece predominantemente de forma não intencional, conforme relatado por Cardoso e Gomes (2011), em períodos de intervalos. As situações de interação dos docentes de modo intencional ocorrem em momentos pontuais como reuniões ou treinamentos. Os servidores técnicos-administrativos têm uma relação de trabalho mais próxima no dia a dia, na qual os conhecimentos do grupo são compartilhados de modo intencional e não intencional com mais frequência.

O efeito da percepção da aprendizagem diminui conforme aumenta a abrangência. Desta forma, as menores médias, tanto dos docentes $(3,81)$ como os técnicos-administrativos $(3,59)$, estão relacionadas com a aprendizagem organizacional. Esta situação evidencia a dificuldade de compartilhamento e gerenciamento do conhecimento entre as diversas áreas da instituição, assim como entre as categorias funcionais docente e técnico-administrativo. Também pode ocorrer devido a fragilidade de métodos organizacionais de sistematização e comunicação do conhecimento adquirido no nível individual e de grupo.

$\mathrm{Na}$ Tabela 3 observam-se as frequências da percepção dos processos de gestão do conhecimento, em que se nota que a socialização é o processo menos percebido pelos respondentes. 
Tabela 3 . Frequências de percepção dos processos de GC como facilitadores da aprendizagem

\begin{tabular}{|l|c|c|c|c|c|c|c|c|c|c|}
\hline \multicolumn{1}{|c|}{ Escala } & $\mathbf{1}$ & $\mathbf{2}$ & $\mathbf{3}$ & $\mathbf{4}$ & $\mathbf{5}$ & $\mathbf{6}$ & $\mathbf{7}$ & Discordo & Neutro & Concordo \\
\hline Externalização & 5 & 12 & 26 & 130 & 234 & 365 & 256 & $\mathbf{4 , 2}$ & $\mathbf{1 2 , 6}$ & $\mathbf{8 3 , 2}$ \\
\hline Combinação & 17 & 26 & 51 & 120 & 156 & 309 & 349 & $\mathbf{9 , 1}$ & $\mathbf{1 1 , 7}$ & $\mathbf{7 9 , 2}$ \\
\hline Socialização & 191 & 188 & 168 & 238 & 131 & 64 & 48 & $\mathbf{5 3 , 2}$ & $\mathbf{2 3 , 2}$ & $\mathbf{2 3 , 6}$ \\
\hline Internalização & 32 & 54 & 52 & 142 & 147 & 215 & 129 & $\mathbf{1 7 , 9}$ & $\mathbf{1 8 , 4}$ & $\mathbf{6 3 , 7}$ \\
\hline
\end{tabular}

Fonte: dados da pesquisa.

Nota-se que os processos de externalização e internalização são percebidos na instituição estudada, com 83,2\% e $63,7 \%$ de concordância respectivamente. Estes resultados vão ao encontro da percepção da aprendizagem individual, pois esta é dependente dos processos acima, nos quais ocorre a conversão do conhecimento tácito para o explícito e vice-versa (NONAKA; TAKEUCHI, 1997; SABHERWAL; BECERRA-FERNANDEZ, 2003). A externalização é mais percebida que a internalização, o que pode levar a suposição de que os colaboradores possuem oportunidades de converterem seus conhecimentos tácitos em explícitos, porém podem ter dificuldades de obter os conhecimentos explícitos disponíveis na organização.

Quanto ao processo de combinação, é o segundo processo melhor percebido, com a frequência de 79,2\% de concordância na percepção dos respondentes. Este processo, segundo Sabherwal e Becerra-Fernandez (2003) colabora para a aprendizagem organizacional, mas os resultados da pesquisa apontam que apesar de perceptível não estão conseguindo atingir o objetivo de proporcionar a aprendizagem adequada para a instituição.

A socialização foi o único processo que obteve a frequência de discordância maior que a de concordância, com $53,2 \%$ contra 23,6\%. Segundo Sabherwal e Becerra-Fernandez (2003) a socialização desempenha relevante papel na transição do conhecimento individual para o grupo, por depender da proximidade física e ação conjunta. Porém, apesar da percepção da eficácia da aprendizagem em grupo estar presente, a socialização não está sendo percebida como facilitadora desta transição. Infere-se que outro processo de gestão do conhecimento possa estar influenciando este aspecto.

$\mathrm{Na}$ segunda etapa foi analisado o modelo de equações estruturais, o que foi possível explicar algumas das correspondências identificadas na análise de frequência. O modelo inicialmente utilizado foi o de Sabherwal e Becerra-Fernandez (2003), conforme já demonstrado na figura 3. A pesquisa realizada pelos autores confirmou as hipóteses H1, H2, H4, H5, H7 e H9. As hipóteses H3, H6 e H8 H9 não foram confirmadas. Optou-se por aplicar o modelo original na IES pesquisada para compreender a percepção dos colaboradores quanto aos processos de gestão do conhecimento.

Embora o modelo utilizado seja validado por Sabherwal e Becerra-Fernandez (2003), os indicadores mostraram que de acordo com os dados coletados pela presente pesquisa precisariam passar por adequação. Assim, aplicouse o Alfa de Cronbrach às dimensões para averiguação da confiabilidade das respostas.

Tabela 4 . Confiabilidade dos dados obtidos

\begin{tabular}{|l|c|c|c|}
\hline \multicolumn{1}{|c|}{ Dimensão } & Alfa de Cronbach & $\begin{array}{c}\text { Alfa de Cronbach } \\
\text { padronizado }\end{array}$ & Número de Itens da Dimensão \\
\hline Aprendizagem individual & 0,856 & 0,856 & 3 \\
\hline Aprendizagem de grupo & 0,853 & 0,854 & 3 \\
\hline $\begin{array}{l}\text { Aprendizagem } \\
\text { organizacional }\end{array}$ & 0,924 & 0,924 & 4 \\
\hline Externalização & 0,872 & 0,874 & 4 \\
\hline Combinação & 0,777 & 0,810 & 3 \\
\hline
\end{tabular}




\begin{tabular}{|l|c|c|c|}
\hline \multicolumn{1}{|c|}{ Dimensão } & Alfa de Cronbach & $\begin{array}{c}\text { Alfa de Cronbach } \\
\text { padronizado }\end{array}$ & Número de Itens da Dimensão \\
\hline Socialização & 0,640 & 0,668 & 4 \\
\hline Internalização & 0,758 & 0,769 & 3 \\
\hline Todas as dimensões & 0,908 & 0,912 & 24 \\
\hline
\end{tabular}

Fonte: dados da pesquisa.

A Tabela 4 apresenta a confiabilidade dos dados indicando que todos os valores foram acima de 0,6 , considerado confiável conforme Hair Jr. et al. (2005). Salienta-se que estes valores foram significantes no nível de 0,01. Desta forma, após três iterações realizadas por meio do módulo AMOS, obteve-se o modelo considerado mais adequado, cujos indicadores ficaram nos limites considerados adequados pela literatura.

Tabela 5 . Indicadores de confiabilidade dos modelos de equações estruturais

\begin{tabular}{|l|c|c|c|}
\hline \multicolumn{1}{|c|}{ Dimensão } & Modelo Inicial & Modelo reespecificado & Valores de referência \\
\hline CFI & 0,875 & 0,952 & $>0,9$ \\
\hline TLI & 0,856 & 0,939 & $>0,9$ \\
\hline CMIN/DF & 3,217 & 2,447 & $<5$ \\
\hline RMSEA & 0,093 & 0,075 & $0,05<\mathrm{x}<0,08$ \\
\hline AGFI & 0,764 & 0,858 & $>0,9$ \\
\hline
\end{tabular}

Fonte: dados da pesquisa.

A figura 4 traz o modelo final considerado para a análise. Este modelo, assim como Sabherwal e Becerra-Fernandez (2003), confirma as hipóteses H1, H2, H5 e H7 e não confirma as hipóteses H3, H6 e H8. Porém, divergindo dos autores, rejeita as hipóteses H4 e H9 e considera novas relações denominadas de H10, H11 e H12.

Figura 4 . Modelo de equações estruturais reespecificado

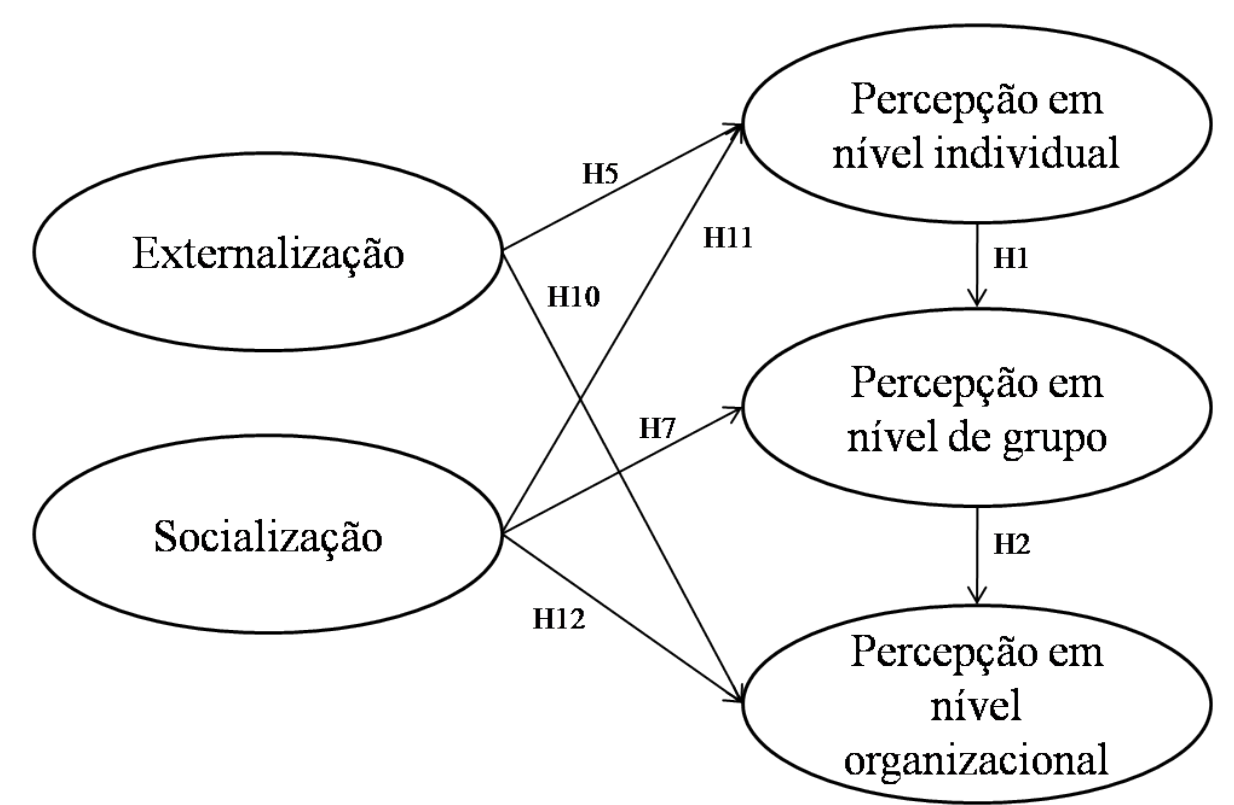

Fonte: Dados da pesquisa. 
Observa-se que na dimensão ontológica, na qual inicialmente desenvolveram-se as hipóteses $\mathrm{H} 1, \mathrm{H} 2$ e H3, a percepção de aprendizagem é originada em nível individual, transferida para o grupo e para a organização. Este modelo reflete o que foi apresentado pela tabela 2, cujas médias obtidas para estes três atributos foram: individual $(5,0)$, em grupos $(4,8) \mathrm{e}$ organizacional $(3,7)$, confirmando as hipóteses H1 e H2. Observa-se que a transmissão do conhecimento do nível individual para o grupo está acontecendo, apesar da diminuição da percepção. Segundo Fialho et al. (2010) este é um processo de evolução nas organização, nas quais as ações convergem para o aprendizado em grupo e deste para a organização.

Para Zaginski, Lima e Costa (2009) compreender o processo de aprendizagem organizacional é um fator relevante para a obtenção de vantagens competitivas. Uma das dificuldades está em localizar as falhas no entendimento da essência do processo de criação do conhecimento, e a compreensão entre a sua relação com a aprendizagem organizacional é considerada a chave para o desenvolvimento do processo de criação e formação de competências. A H3 não foi confirmada revelando que as inter-relações entre indivíduos, o grupo e a organização reforçam a visão de que a agregação de conhecimento para os níveis com maior coletividade é relevante para o crescimento do conhecimento (SABHERWAL; BECERRAFERNANDEZ, 2003). Esta situação evidencia que individualmente a aprendizagem de cada funcionário não gera impacto significativo na aprendizagem organizacional, o que denota que é necessária maior abertura para que os indivíduos possam expressar seu conhecimento de forma sistematiza no intuito de colaborar com o coletivo.

Conforme Nonaka e Takeuchi (2008) o processo de criação do conhecimento inicia-se pela socialização, passando pela externalização, combinação e internalização. $\mathrm{Na}$ dimensão epistemológica ocorre a interação entre conhecimento tácito e explícito, porém ocorre no sentido tácito para tácito e explícito, mas não no explícito para explícito e tácito. Esta constatação permite inferir que há a geração de conhecimento, porém os fluxos podem sinalizar que a espiral é inicializada, na qual o conhecimento tácito é socializado e posteriormente explicitado, mas é interrompida na transmissão de conhecimento explícito para explícito e posteriormente para tácito. Infere-se que a comunicação e formalização dos procedimentos, normas e regulamentos disponíveis a toda organização não está sendo realizada de forma efetiva, a fim de que sejam internalizadas por todos os colaboradores de forma institucionalizada.

Estes resultados podem estar relacionados com a população e a amostra coletada, que segundo Silva (1991), as IES possuem um profissional diferenciado das demais organizações e desenvolve um estilo próprio de estrutura. Aproximadamente 56,7\% dos colaboradores são docentes, que tem a oralidade como uma das principais ferramentas para a execução de suas atividades acadêmicas. Outra característica muito utilizada é a preparação de materiais didáticos. Estas características estão fortemente ligadas aos processos de socialização e externalização.

$\mathrm{Na}$ dimensão epistemológica foram desenvolvidas inicialmente as hipóteses $\mathrm{H} 4, \mathrm{H} 5, \mathrm{H} 6, \mathrm{H} 7, \mathrm{H} 8$ e H9. Destas somente as hipóteses $\mathrm{H} 5$ e $\mathrm{H} 7$ foram confirmadas e as hipóteses H10, H11 e H12 foram percebidas após a pesquisa.

Para a aprendizagem individual somente a $\mathrm{H} 5$ foi confirmada, demonstrando que o processo de exteriorização facilita a aprendizagem. A H4 não foi confirmada, na qual supunha-se que a internalização facilitaria a aprendizagem individual. Segundo Sabherwal e Becerra-Fernandez (2003) os processos de internalização e de exteriorização ajudam a "destravar" o conhecimento detido pelo indivíduo, no qual a internalização está relacionada à aprendizagem e a externalização é essencial para a articulação do conhecimento.

Percebe-se que a articulação do conhecimento está ocorrendo, porém a operacionalização dos conhecimentos organizacionais pode estar prejudica, pois os colaboradores não percebem este processo como facilitador da aprendizagem individual. No entanto, os indivíduos aprendem, e infere-se que esta aprendizagem pode ocorrer por meio do ambiente interorganizacional, cuja influência não foi estudada pela pesquisa. Assim, o conhecimento adquirido pelos colaboradores fora dos limites da instituição pode ser socializado e posteriormente externalizado. Este tipo de interação de conhecimento pode ser benéfica, pois engloba todo o contexto no qual a IES está inserida, mas necessitaria da combinação dos conhecimento internos para gerar vantagem competitiva.

$\mathrm{Na}$ aprendizagem em grupo somente a $\mathrm{H} 7$ foi confirmada expondo que somente o processo de socialização facilita a aprendizagem. As hipóteses H6 e H8, também relacionadas à aprendizagem em nível de grupo não foram confirmadas. Infere-se que 
a hipótese H6 não ter sido confirmada pode estar relacionada ao fato de a organização não ter uma cultura consolidada de documentação e registro de procedimentos.

A percepção dos colaboradores de que a combinação facilita a aprendizagem organizacional não foi confirmada (H8), apesar da frequência de concordância ter sido alta. Apesar do caráter de comunicação e transparência de toda organização pública, observase que a publicação de portarias, resolução, pareceres pode não ser suficiente para completar o ciclo de criação do conhecimento, pois de acordo com Nonaka e Takeuchi (2008), a mera combinação de partes diferentes de informações explícita em um novo todo não amplia, necessariamente, a base de conhecimentos existentes na organização. Esta interrupção na espiral do conhecimento revela o que Von Krogh, Ichijo e Nonaka (2001) e Ichijo (2008) constataram em suas pesquisas, de que a gestão do conhecimento não é um processo fácil de ser percebido e implementado, pois ocorrem barreiras individuais e organizacionais que impedem o seu fluxo contínuo.

Observa-se que os processos dos quais é necessária a documentação do conhecimento (H6, H8 e H9) não são percebidos pelos respondentes. Deduz-se que documentar o conhecimento demanda de muito tempo e planejamento tanto para a execução quanto para a comunicação, o que pode estar interferindo nesta etapa do processo. No entanto, a socialização mostrou-se um caminho que pode influenciar a aprendizagem em grupo, conforme hipótese (H7) também confirmada por Sabherwal e BecerraFernandez (2003).

No nível da aprendizagem organizacional a hipótese H9 não foi confirmada, na qual a combinação facilita a aprendizagem. Por sua vez, as hipóteses H10 e H12 foram desenvolvidas após a pesquisa. Conforme se pode observar em Machado e Silveira (1998), os grupos deliberativos e executivos de uma universidade são uma composição heterogênea, contando com representantes docentes, discentes, técnico-administrativos e comunidade externa, o que pode explicar alguns fenômenos revelados pela pesquisa. A característica de tomada de decisão colegiada e participativa pode explicar as novas relações observadas.

A H10 relaciona a externalização à aprendizagem organizacional. Geralmente esta ferramenta está relacionada à aprendizagem em nível individual (NONAKA; TAKEUCHI, 1997; SABHERWAL;
BECERRA-FERNANDEZ,2003), porém novamente as características docentes podem produzir resultados diferentes numa IES, divergindo do que preconiza a literatura. A familiarização pelos colaboradores com as atividades didáticas e pedagógicas, a produção acadêmica, as quais são muito documentadas, podem interferir diretamente na aprendizagem organizacional, já que muitas vezes esta documentação é um trabalho solitário e não coletivo. Outra inferência que se pode fazer para explicar esta relação pode ser associada à forma colegiada e participativa com que são tomadas as decisões. Ocorrem muitos debates nos conselhos, nos quais a externalização de argumentos dos participantes podem influenciar diretamente a aprendizagem de toda a organização.

Porém na pesquisa realizada, tanto a aprendizagem individual e organizacional (H11 e H12) sofrem sua influência. O conhecimento é compartilhado por este processo, que é o gatilho para a dinâmica da espiral do conhecimento. Diversos docentes assumem responsabilidades de gestão e acabam adotando as práticas anteriormente descritas para a gestão da IES, devido à familiarização com as mesmas. Os docentes, por participarem de diversos grupos e terem sua carga de trabalho fragmentada entre atividades acadêmicas e de gestão, acabam por socializar seus conhecimentos com diversos grupos e desta forma, a socialização pode impactar diretamente na aprendizagem organizacional sem necessariamente passar pelos grupos, pois permeia grupos heterogêneos, diferindo do que diz a literatura quando tratada em uma organização empresarial. No entanto, estes conhecimentos obtidos não estão sendo convertidos em nível organizacional, de forma de seja institucionalizado, formalizado e utilizado para incrementar a espiral do conhecimento.

A aprendizagem organizacional é construída por meio da aprendizagem dos diversos grupos que a compõe, que por sua vez traz a bagagem dos indivíduos ali inseridos. Por meio da modelagem de equações estruturais buscou-se entender como a relação entre as dimensões ontológica e epistemológica acontecem em uma instituição de nível superior, especificamente do Estado de Santa Catarina. Observou-se que o compartilhamento desta IES acontece de forma divergente de outros tipos de organização. Percebe-se que o processo de socialização permeia os três níveis de aprendizagem, o qual se torna importante para a evolução da espiral do conhecimento. Por outro lado, quando é necessária a conversão do conhecimento explícito, os processos de internalização e combinação não são percebidos. 


\section{Conclusão}

As IES são reconhecidas por sua capacidade de criação, armazenamento e disseminação de conhecimento por meio das atividades de ensino, pesquisa e extensão. No entanto, os processos administrativos, e de gestão, são fontes relevantes para conhecer como estas instituições estabelecem e propagam o conhecimento, no intuito de aprimorar suas atividades e enriquecer o repertório disponível a seus colaboradores.

Percebeu-se nas bases de dados pesquisadas, que o tema gestão do conhecimento, quando abordado em IES, refere-se à relação professor-aluno, porém a disseminação do conhecimento entre seus colaboradores, sejam eles docentes ou técnicoadministrativos, não foi encontrada. Uma universidade, considerada uma organização complexa, cujo objetivo é propriamente a disseminação e a geração do conhecimento, tem em seu capital humano uma fonte rica para a melhoria de seus processos gerenciais. Saber armazenar e utilizar o conhecimento gerado para a solução de seus problemas se torna relevante para colocar em prática o que a teoria argumenta.

Constatou-se que na dimensão ontológica a percepção de aprendizagem é originada em nível individual, transferida para o grupo e para a organização. Foi possível perceber que conforme aumenta o nível da aprendizagem vai se perdendo a percepção da capacidade de facilitação da aprendizagem. A aprendizagem individual apresentou a maior frequência de percepção de eficácia dos processos de gestão do conhecimento, seguida da de grupo e da organizacional.

Na dimensão epistemológica a espiral do conhecimento é iniciada, na qual o conhecimento tácito é convertido em tácito e explicito pelos processos de socialização e externalização, mas os processos subsequentes de combinação e internalização não foram correlacionados com os níveis de aprendizagem. Este resultado pode ser resultado da população e da amostra coletada, pois docentes utilizam a oralidade e a escrita como ferramentas de trabalho, o que pode refletir para os demais colaboradores da instituição.

A aprendizagem individual foi obtida pelo processo de externalização, no qual o conhecimento é convertido do tácito para o explícito. Infere-se que este processo de gestão do conhecimento satisfaz ao objetivo de aprendizagem individual, contudo a internalização não possui forte correlação.
A socialização proporciona a aprendizagem em grupo, conforme a literatura sobre o tema preconiza, porém a IES pesquisada também proporciona a aprendizagem de grupo e organizacional. Observando a estrutura como são compostas estas instituições, pode-se supor que a heterogenia de grupos que tomam decisões colegiadas, e o fato do docente ter uma carga de trabalho dividida entre atividades acadêmicas e de gestão, a socialização facilita a transição do conhecimento de forma flexível permeando todos os níveis da aprendizagem.

Apesar do processo de combinação ser percebido pelos colaboradores, não apresentou correlação forte com a aprendizagem organizacional. A combinação gera o conhecimento sistematizado e com o realinhando deste processo pode ser possível institucionalizar o conhecimento criado, para além de repositório de documentos e possibilitar a realimentação da espiral do conhecimento.

Por meio deste estudo foi possível perceber que o processo de gestão do conhecimento pode ocorrer de formas diversas, variando de acordo com o tipo de organização. No caso específico da IES investigada neste estudo, o processo de socialização ocorre nos três níveis de aprendizagem, tornando-se significativo para a evolução da espiral do conhecimento. Sendo assim, o estudo contribui para novos avanços nesta área de estudo.

Conclui-se que o conhecimento é gerado e socializado nos níveis de aprendizagem, porém a sistematização dos conhecimentos para serem utilizados na consecução dos objetivos da instituição necessita de adequação para facilitar a aprendizagem organizacional.

Como contribuição prática, o presente estudo mostra uma "fotografia" da situação atual da instituição. A partir dela é possível vislumbrar as potencialidades e fragilidades da instituição e ser o ponto inicial para o encaminhamento de um projeto. Como contribuição teórica, buscou-se relacionar dois temas transdisciplinares, além da preocupação nos estudos organizacionais. Como sugestão propõe-se outros estudos em IES, com a finalidade de sinalizar outras perspectivas de gestão do conhecimento para IES, por estas serem consideradas organizações complexas com características específicas, que as diferem das organizações empresariais. 


\section{Referências}

AMAYAH, A. T. Determinants of knowledge sharing in a public sector organization. Journal of Knowledge Management, v. 17, n. 3, p. 454-471, 2013.

ARGOTE, L.; MIRON-SPEKTOR, E. Organizational learning: from experience to knowledge. Organization Science, v. 22, n. 5, p. 1123-1137, 2011.

BATISTA, F. F. Modelo de gestão do conhecimento para a administração pública brasileira: como implementar a gestão do conhecimento para produzir resultados em benefício do cidadão. Brasília: Ipea, 2012.

BRASIL. Lei $\mathbf{n}^{\circ} \mathbf{5 . 5 4 0}$, de 28 de novembro de 1968, fixa normas de organização e funcionamento do ensino superior e sua articulação com a escola meia e da outras providências. Disponível em: <http:/ / www.planalto.gov.br/ccivil_03/leis/15540.htm>. Acesso em: 11 maio 2014.

Lei $\mathbf{n}^{\circ} \mathbf{9 . 3 9 4}$, de 20 de dezembro de 1996, estabelece as diretrizes e bases da educação nacional. Disponível em: < http://portal.mec.gov.br/ arquivos/pdf/ldb.pdf $>$. Acesso em: 11 maio 2014.

CAJUEIRO, Joyce L. G.; SICSÚ, Abraham B.; RIBEIRO, Ana Regina B. Elementos preliminares para a construção de modelos: a contribuição da gestão do conhecimento para Instituições de ensino superior. Revista Gestão Industrial, v. 05, n. 03, p. 219-234, 2009.

CARDOSO, L.; GOMES, D. Knowledge

Mangement and Innovation: mapping the use of knowledge in organizations. In: Mesquita, A. Technology for creativity and innovation: tools, techniques and applications, p. 237-266, 2011.

CARDOSO, Olinda N. P.; MACHADO, Rosa T. M. Gestão do conhecimento usando data mining: estudo de caso na Universidade Federal de Lavras. Revista de Administração Pública, v. 42, n. 3, maio/jun, p. 495-528, 2008.

CHOO, C. W. A Organização do Conhecimento. São Paulo: Editora Senac, 2003.

COHEN, Wesley M.; LEVINTHAL, Daniel A. Absorptive capacity: a new perspective on learning and innovation. Administrative Science Quaterly, v. 35, n.1, p. 128-152, 1990.
CRESWELL, John W. Projeto de pesquisa: métodos qualitativo, quantitativo e misto. 2. ed. Porto Alegre: Artmed, 2007.

DAVENPORT, Thomas H.; PRUSAK, Laurence. Conhecimento empresarial: como as organizações gerenciam o seu capital intelectual. 4. ed. Rio de Janeiro: Campus, 1998.

ETZIONI, A. Humble decision making. Harvard Business Review, v. 67, n. 4, p. 122, 1989.

FIALHO, Francisco Antônio Pereira; MACEDO, Marcelo; SANTOS, Neri dos; MITIDIERI, Tibério da Costa. Gestão do conhecimento e aprendizagem: as estratégias competitivas da sociedade pós-industrial. Florianópolis: Visual Books, 2006.

Gestão do conhecimento

organizacional. Florianópolis: Editora da UFSC, 2010.

HAIR JR, Joseph F. et al. Fundamentos de métodos de pesquisa em administração. Porto Alegre: Bookman, 2005.

HARTUNG, K.; OLIVEIRA, M. Communities of practice: creating and sharing knowledge. Revista de Gestão, v. 20, n. 3, p. 407-422, 2013.

HE, H.; BARUCH, Y.; LIN, C. P. Modeling team knowledge sharing and team flexibility: the role of within-team competition. Human Relations, v. 67, n. 8, p. 947-978, 2014.

HEIZIG, P. Harmonisation of knowledge management: comparing $160 \mathrm{KM}$ frameworks around the globe. Journal of Knowledge Management, v. 13, n. 4, p. 4-31, 2009.

ICHIJO, Kazuo. Da administrção à promoção do conhecimento. In: TAKEUCHI, Hirotaka; NONAKA, Ikujiro. Gestão do Conhecimento. Porto Alegre: Bookman, 2008.

ISIDRO-FILHO, A. I. Mecanismos de Aprendizagem em Organizações: Desenvolvimento e Validação de uma Escala de Medida. Revista de Administração Mackenzie, v. 10, n. 1, p. 37-57, 2009.

MACHADO, Nelson Santos. SILVEIRA, Amélia. Configurações estruturais em organizações universitárias. Florianópolis: Insular, 1998. 
MEYER JUNIOR, Victor. A escola como organização complexa. In: EYNG, Ana Maria; GISI, Maria Lourdes. Políticas e gestão da educação superior. Ijuí. Editora UNIJUI, 2007.

NONAKA, I.; TAKEUCHI, H. The knowledgecreating company: how japanese companies create the dynamics of innovation? Oxford: Oxford University Press, 1995.

NONAKA, Ikujiro. A empresa criadora do conhecimento. In HOWARD, Robert. Aprendizado organizacional: gestão de pessoas para a inovação contínua. Trad. Bazán Tecnologia e Lingüística. Rio de Janeiro: Campus, 2000.

. Teoria da criação do conhecimento organizacional. In: TAKEUCHI, Hirotaka; NONAKA, Ikujiro. Gestão do Conhecimento. Porto Alegre: Bookman, 2008.

; TAKEUCHI, Hirotaka. Criação de conhecimento na empresa. Rio de Janeiro: Campus, 1997.

PEREIRA, Mauricio F. et.al. A história e a experiência da Universidade Federal de Santa Catarina com o processo de planejamento estratégico. 2008. In: DOMINGUES, Maria José C. S.; SILVEIRA, Amélia. Gestão de ensino superior: temas contemporâneos. Blumenau: Edifurb, 2008.

POKHAREL, M. P.; CHOI, S. A. Exploring the relationships between the learning organization and organizational performance. Management Research Review, v. 38, n. 2, p. 126-148, 2015.

RAMAYAH, T.; YEAP, J. A. L.; IGNATIUS, J. Assessing knowledge sharing among academics: a validation of the knowledge sharing behavior scale (KSBS). Evaluation Review, v. 38, n. 2, p. 160-187, 2014.

SABHERWAL, Rajiv; BECERRA-FERNANDEZ, Irma. An empirical study of the effect of Knowledge management processes at individual, group, and organizational levels. Decision Sciences, v. 34, n. 2, p. 225-60, 2003.

SANCHEZ, Ron; HEENE, Aime. A Competence perspective on strategic learning and knowledge management. In: SANCHEZ, R.; HEENE, A. (Eds.). Strategic learning and knowledge management. Chichester: John Wiley \& Sons, p. 3-15, 1997.
SILVA, Clóvis Machado da. Modelos Burocrático

e Político de Estrutura Organizacional de Universidades. In: Temas de administração universitária. Florianópolis/CPGA, 1991.

TAKEUCHI, Hirotaka; NONAKA, Ikujiro. Criação e dialética do conhecimento. In: TAKEUCHI, Hirotaka; NONAKA, Ikujiro. Gestão do

Conhecimento. Porto Alegre: Bookman, 2008.

TERRA, J. C. C. Gestão do Conhecimento: aspectos conceituais e estudo exploratório sobre as práticas de empresas brasileiras. In Fleury, M. T. L. \& Oliveira Jr., M. M. (Orgs.). Gestão estratégica do conhecimento: integrando aprendizagem, conhecimento e competências. São Paulo: Atlas, 2001.

TERRA, José Cláudio Cyrineu. Gestão do conhecimento: o grande desafio empresarial. 4. ed. São Paulo: Negócio Editora, 2005.

VON KROGH, Georg; ICHIJO, Kazuo; NONAKA, Ikujiro. Facilitando a criação do conhecimento: reinventando a empresa com o poder da inovação contínua. Rio de Janeiro: Campus, 2001.

ZANGISKI, Marlene A. S. G.; LIMA, Edson P.; COSTA, Sérgio E. G. Aprendizagem organizacional e desenvolvimento de competências: uma síntese a partir da gestão do conhecimento. Produto e Produção, v. 10, n. 1, p. 54-74, 2009. 